\title{
The association among MDCT-derived three-dimensional visceral adiposities on cardiac diastology and dyssynchrony in asymptomatic population
}

\author{
Yau-Huei Lai ${ }^{1,2,3,4 \dagger}$, Charles Jia-Yin Hou ${ }^{1,2,3,4+}$, Chun-Ho Yun ${ }^{5,6}$, Kuo-Tzu Sung 1,2,3,4, Cheng-Huang Su, 1,2,3,4, \\ Tung-Hsin Wu ${ }^{6}$, Fei-Shih Yang ${ }^{5}$, Ta-Chuan Hung ${ }^{1,2,3,4,7}$, Chung-Lieh Hung ${ }^{1,2,3,4,7^{*}+}$, Hiram G. Bezerra ${ }^{8}$ \\ and Hung-I Yeh ${ }^{1,2,3,4}$
}

\begin{abstract}
Background: Visceral adipose tissue, a biologically active fat depot, has been proposed as a reliable marker for visceral adiposity and metabolic abnormalities. Effects of such adiposity on LV diastolic function and dyssynchrony remained largely unknown.

Methods: We assessed pericardial fat (PCF) and thoracic peri-aortic fat (TPAF) by three-dimensional (3D) volume-vender multi-detector computed tomography (MDCT) (Aquarius 3D Workstation, TeraRecon, San Mateo, CA, USA). Echo-derived diastolic parameters and tissue Doppler imaging (TDI) defined mitral annular systolic ( $\mathrm{S}^{\prime}$ ), early diastolic (E') velocities as well as LV filling (E/E') were all obtained. Intra-ventricular systolic (Sys-D) and diastolic (Dias-D) dyssynchrony were assessed by TDI method.
\end{abstract}

Results: A total of 318 asymptomatic subjects (mean age: 53.5 years, $36.8 \%$ female) were eligible in this study. Greater PCF and TPAF were both associated with unfavorable diastolic indices and higher diastolic dyssynchrony (all $p<0.05)$. These associations remained relatively unchanged in multi-variate models. PCF and TPAF set at 81.68 \& $8.11 \mathrm{ml}$ yielded the largest sensitivity and specificity (78.6 and $60 \%$ for PCF, 75 and $66.6 \%$ for TPAF, respectively) in predicting abnormally high LV diastolic dyssynchrony, which was defined as Dias- $\mathrm{D} \geqq 55 \mathrm{~ms}$.

Conclusion: Increasing visceral adiposity may be associated with adverse effects on myocardium, primarily featured by worse diastolic function and greater degree of dyssynchrony.

Keywords: Pericardial fat, Diastolic dysfunction, Dyssynchrony, Tissue Doppler

\section{Background}

Heart failure (HF) has emerged as a rapid growing epidemic in recent years [1]. There has been increasing academic interest in heart failure with preserved ejection fraction (HFpEF), which may account for nearly half of the HF population [2]. Recent studies have shown that mechanical dyssynchrony, an important pathophysiologic

\footnotetext{
* Correspondence: jotaro3791@gmail.com

${ }^{\dagger}$ Equal contributors

'Division of Cardiology, Department of Internal Medicine, Mackay Memorial Hospital, No. 92, Sec. 2, Zhongshan N. Road, Taipei, Taiwan

${ }^{2}$ Division of Cardiology, Department of Internal Medicine, Mackay Memorial Hospital, Hsinchu, Taiwan

Full list of author information is available at the end of the article
}

surrogate associated with myocardial asynergy, is not solely limited to systolic heart failure, but also exists in HFpEF and normal QRS duration [3, 4]. Diastolic dysfunction and its progression are independent predictors of incident heart failure [5].

Accumulating epidemiologic data have recently suggested that metabolic abnormalities accompanied by obesity, excessive body fat and systemic inflammation can confer higher heart failure risks [6-9], partially through left ventricular (LV) remodeling or hypertrophy [10, 11]. Obesity is associated with altered LV remodeling, possibly due to increased hemodynamic load, neurohormonal activation, and increased cytokine production [12]. A recent 
study showed that overweight and obesity were independent predictors of LV diastolic dysfunction [13]. However, the mechanistic link between excessive visceral adiposity and LV mechanical asynergy, especially diastolic dysfunction, remained largely unexplored.

Pericardial fat (PCF), which can be accurately quantified by various imaging tools and serve as a reliable marker of visceral adiposity $[14,15]$, has been recently proposed as an active source of proatherogenic cytokines that mediate systemic inflammation and metabolic derangements in obese subjects [16]. It had also been shown to influence LV structure and function through mechanical or paracrine effects, partially due to its anatomic proximity to the myocardium [17]. We sought to investigate the association between visceral adiposity and LV diastolic indices in subjects undergoing cardiovascular health survey.

\section{Methods}

\section{Subjects}

From 2009 to 2013, we consecutively studied subjects who attended primary cardiovascular health survey in a tertiary medical center in Taipei, Taiwan. Participants with decompensated heart failure, unstable coronary events, symptomatic angina or those who underwent hemodialysis were precluded from this primary screen program. Detailed physical examination was performed as well as a thorough review of baseline characteristics, medical history including current smoking behavior and regular physical activity from structured questionnaires. Subjects with known cardiovascular disease history defined as previous myocardial infarction, coronary arterial disease, stroke, prior hospitalization for congestive heart failure and peripheral arterial disease were excluded. Subjects with history of atrial fibrillation, left bundle branch block or pacemaker implantation were also excluded. A total of 318 subjects after exclusion during study period were finally enrolled.

Hypertension was defined as systolic blood pressure higher than $140 \mathrm{mmHg}$, diastolic blood pressure higher than $90 \mathrm{mmHg}$ or previously diagnosed hypertension under medication control. Diabetes was defined as fasting glucose level more than $126 \mathrm{mg} / \mathrm{dL}$ or previously diagnosed diabetes under medication control. Hyperlipidemia was defined as those with known history and/or who use lipidlowering drugs, such as statins or fibrates, on a daily basis.

This study has been approved by Mackay Memorial Hospital Institutional Review Board with adherence to research ethics (IRB number: 14MMHIS161). All participants were adults and provided written informed consent to be included in the study.

\section{Lab data acquisition and analysis}

Hitachi 7170 Automatic Analyzer (Hitachi Corp. Hitachinaka Ibaraki, Japan) was used to measure fasting and postprandial glucose levels (hexokinase method), HbA1c, creatinine (kinetic colorimetric assay), total cholesterol and triglyceride (TG). Lipid profiles including low-density lipoprotein (LDL) and high-density lipoprotein cholesterol (HDL) were obtained by homogenous enzymatic colorimetric assay. High-sensitivity CRP (hs-CRP) levels were determined by using a highly sensitive, latex particle-enhanced immunoassay Elecsys 2010 (Roche, Mannheim, Germany).

\section{Echocardiographic assessment}

Each subject underwent two-dimensional and color Doppler transthoracic echocardiogram (GE, Vivid 7, Vingmed Ultrasound, Norway) equipped with 2-4 MHz transducer at left decubitus position. Standardized echocardiography imaging protocol including measurement of left atrial dimension (LAD), wall thickness, LV volumes (biplane Simpson method), and LV mass (American Society of Echocardiography criteria) [18] were all obtained. LV diastolic function was determined by pulsed-wave Doppler of transmitral inflow early (E) and late diastolic (A) LV filling velocities measured at the tip of the mitral leaflets in the apical 4-chamber view. Diastolic functional parameters including early mitral inflow velocity (E), early-to-late inflow ratio (E/A), deceleration time (DT), isovolumetric relaxation time (IVRT), and spectral tissue Doppler imaging (TDI) defined mitral annulus systolic ( $\mathrm{S}^{\prime}$ ) and early diastolic (E') velocities were all obtained. The average values of S' and E' at LV basal lateral and septal segments were presented throughout this manuscript. Grading of diastolic dysfunction was then determined according to EAE/ASE recommendations [19].

For pulsed wave TDI-based measures, highest temporal resolution with frame rates $>100$ frames/sec with caution to optimize the parallel alignment for TDI tracing with myocardial longitudinal motion was achieved for raw imaging recording for subsequent dyssynchrony analysis. Intra-ventricular horizontal dyssynchrony was presented as the absolute time-to-peak velocity difference of $S^{\prime}$ and E' (Sys-D, Dias-D) between LV basal lateral and septal segments from apical 4-chamber views (Additional file 1: Figure S1). Significant diastolic dyssynchrony was defined as Dias- $\mathrm{D} \geqq 55 \mathrm{~ms}$ based on a previous article [20].

\section{Measurement of pericardial fat}

Multidetector computed tomography (MDCT) study was performed by a 16-slice scanner (Sensation 16, Siemens Medical Solutions, Forchheim, Germany) with $16 \mathrm{~mm} \times$ $0.75 \mathrm{~mm}$ collimation, rotation time $420 \mathrm{~ms}$ and tube voltage of $120 \mathrm{kV}$. In one breath-hold, images were acquired from above the level of tracheal bifurcation to below the base of heart using prospectively ECG triggering with the centre of the acquisition at $70 \%$ of the R-R interval. From the raw data, the images were reconstructed with standard 
kernel in $3 \mathrm{~mm}$ thick axial, non-overlapping slices and $25 \mathrm{~cm}$ field of view. Pericardial fat (PCF) and thoracic peri-aortic fat (TPAF) were quantified using a dedicated workstation (Aquarius 3D Workstation, TeraRecon, San Mateo, CA, USA). The semi-automated segmentation technique was developed for quantification of fat volumes. We traced the region of interest manually and defined fat tissue as pixels within a window of -195 to $-45 \mathrm{HU}$ and a window centre of $-120 \mathrm{HU}$. PCF was defined as any adipose tissue located within the pericardium from the level of left main coronary artery to the cardiac base (Additional file 2: Figure S2A and 2B). TPAF was defined as all of the adipose tissue surrounding the descending thoracic aorta which extended $67.5 \mathrm{~mm}$ from the level of the bifurcation of pulmonary arteries with cranial-caudal coverage. This approach has previously been validated [21].

\section{Statistical analysis}

Continuous data was expressed as the mean and standard deviation with categorical data expressed as the frequency and proportion of occurrence in all subjects. Differences of baseline demographics, metabolic and echocardiographic parameters between PCF and TPAF tertile groups were tested by ANOVA test with categorical data analyzed by chi-square or Fisher's exact test as appropriate. Uni- and multivariable regression models including confounding variables of age, gender, BMI, blood pressure, LV mass index and clinical histories (hypertension, diabetes, hyperlipidemia, smoking habits) were used to determine the independent association between adipose tissue volumes (both PCF \& TPAF), LV structural/functional parameters and degree of dyssynchrony (Sys-D/Dias-D). The area under the receiveroperator characteristic curve (AUROC) for PCF/TPAF in identifying significant LV septal and lateral wall (systolic and diastolic) timing differences was used as a summary measure of clinical outcome measure for dyssynchrony (defined as $\geqq 60$ \& $55 \mathrm{~ms}$ for systolic and diastolic dyssynchrony, respectively), with corresponding $95 \%$ confidence interval (CI) was further reported.

All data were analyzed using a commercial software STATA 8.2 package (Stata Corp., College Station, Texas). The significance of p level ( $\alpha$-value) for all analysis was two-sided, with a value less than 0.05 considered statistically significant.

\section{Results}

Baseline demographics and the association between visceral adiposity and clinical information

Of all 318 subjects (mean age: 53.5 years, $36.8 \%$ female) enrolled, 102 (32.1\%) had hypertension, 46 (14.5\%) had diabetes, and 109 (34.3\%) had hyperlipidemia with a mean PCF and TPAF volume of $80.6 \pm 33 \& 7.89 \pm$
$4.47 \mathrm{ml}$, respectively. Median PCF volume was $75.98 \mathrm{ml}$ (interquartile range $=39.02$ ), and median TPAF volume was $7.02 \mathrm{ml}$ (interquartile range $=5.08$ ). The association between baseline demographics, biochemical data and visceral adiposity are summarized in the first half of Table 1. In brief, both higher PCF and TPAF were associated with higher systolic blood pressure, male predominance and decreased HDL levels (all $p<0.05$ ). There were also significant differences in age and diastolic blood pressure across PCF tertile groups (both $p<0.05$ ).

\section{The association between visceral adiposity, cardiac geometry and diastolic function}

There were 148 subjects with Grade 1 diastolic dysfunction and 70 subjects with Grade 2 diastolic dysfunction. The association between echocardiographic data and visceral adiposity was summarized in the latter half of Table 1. Across tertile groups, greater PCF and TPAF were both associated with increased LAD, reduced $\mathrm{E}$ and E' velocities, lower E/A ratio and prolonged DT (all $p<0.05$ ). PCF was also associated with elevated E/E' ratio, prolonged IVRT and increased interventricular septum thickness (all $p<0.05$ ). TPAF was associated with significant increases in LV end-systolic volume and both wall thickness (all $p<0.05$ ). There was no significant difference in LV ejection fraction (LVEF) with either adipose tissue. In Table 2, we re-categorized all subjects into with/ without diastolic dysfunction groups according to EAE/ ASE recommendations [19] and compared their key characteristics. There were significant differences in age, gender, both adipose tissue volumes, LA diameter and all Doppler parameters of diastolic function (all $p<0.05$ ). Increased BNP was also borderlinely associated with diastolic dysfunction $(p=0.06)$.

Figure 1a-d demonstrated the linear regression plots of PCF/TPAF with E' and E/E'. Both adipose tissue volumes had excellent correlation with these two key parameters of diastolic dysfunction (decreased E', increased $\mathrm{E} / \mathrm{E}^{\prime}$; all $\left.p<0.001\right)$. In Fig. $2 \mathrm{a}$ and $\mathrm{b}$, receiver-operating characteristic (ROC) analysis showed that both PCF and TPAF had modest area under curve (AUROC) for identifying diastolic dysfunction $(0.712$ \& $0.666,95 \% \mathrm{CI}$ : 0.651-0.773 \& 0.604-0.729 for PCF \& TPAF, respectively, both $p<0.001)$. PCF and TPAF set at $67.31 \&$ $6.69 \mathrm{ml}$ yielded the largest sensitivity and specificity (72.9 and $62 \%$ for PCF, 62.8 and $60 \%$ for TPAF, respectively) in predicting diastolic dysfunction. It should be noted here that the $\mathrm{p}$ values yielded by ANOVA (Table 1) and linear regression analysis (Fig. 1d) between TPAF and E/E' seemed contradictory. This discrepancy might be attributed to non-normal distribution of TPAF.

In Table 3, we showed the uni- and multi-variate regression models regarding the association between visceral adiposity and various echocardiographic parameters. After 
Table 1 Baseline demographics, biomarkers and echocardiographic parameters categorized by pericardial and periaortic fat volumes

\begin{tabular}{|c|c|c|c|c|c|}
\hline \multirow[t]{2}{*}{$\mathrm{PCF}, \mathrm{ml}$} & Q1 $(n=106)$ & Q2 $(n=106)$ & Q3 $(n=106)$ & \multirow[t]{2}{*}{$\mathrm{F}$} & \multirow[t]{2}{*}{$p$-value } \\
\hline & $<63.6 \mathrm{ml}$ & $63.6-88.7 \mathrm{ml}$ & $>88.7 \mathrm{ml}$ & & \\
\hline Age (years) & $51.67 \pm 8.81$ & $53.60 \pm 9.98$ & $55.40 \pm 10.29$ & 3.609 & 0.028 \\
\hline Male & 56 (52.8 \%) & 72 (67.9\%) & 73 (68.9\%) & - & 0.025 \\
\hline $\mathrm{BMI}\left(\mathrm{kg} / \mathrm{m}^{2}\right)$ & $24.78 \pm 3.50$ & $25.48 \pm 3.97$ & $25.40 \pm 3.41$ & 0.787 & 0.456 \\
\hline $\mathrm{SBP}(\mathrm{mmHg})$ & $119.04 \pm 17.68$ & $126.30 \pm 16.88$ & $127.15 \pm 15.20$ & 8.193 & $<0.001$ \\
\hline $\mathrm{DBP}(\mathrm{mmHg})$ & $74.42 \pm 11.17$ & $77.97 \pm 9.99$ & $77.62 \pm 10.27$ & 5.121 & 0.006 \\
\hline HbA1c (\%) & $5.97 \pm 0.75$ & $5.97 \pm 0.62$ & $6.08 \pm 0.73$ & 1.242 & 0.29 \\
\hline Cholesterol (mg/dL) & $201.27 \pm 37.52$ & $203.14 \pm 37.28$ & $205.33 \pm 42.73$ & 0.977 & 0.378 \\
\hline $\mathrm{TG}(\mathrm{mg} / \mathrm{dL})$ & $137.70 \pm 77.21$ & $149.98 \pm 90.00$ & $144.72 \pm 94.28$ & 0.733 & 0.481 \\
\hline $\mathrm{HDL}(\mathrm{mg} / \mathrm{dL})$ & $51.50 \pm 13.96$ & $50.07 \pm 14.13$ & $48.72 \pm 13.67$ & 4.12 & 0.017 \\
\hline LDL (mg/dL) & $130.75 \pm 33.79$ & $132.18 \pm 33.79$ & $135.29 \pm 33.23$ & 1.595 & 0.205 \\
\hline Hs-CRP (mg/dL) & $0.174 \pm 0.022$ & $0.255 \pm 0.035$ & $0.26 \pm 0.043$ & 1.312 & 0.271 \\
\hline $\mathrm{BNP}(\mathrm{pg} / \mathrm{mL})$ & $33.62 \pm 6.33$ & $25.64 \pm 4.23$ & $32.88 \pm 7.19$ & 0.532 & 0.588 \\
\hline Hypertension & $28(26.4 \%)$ & 35 (33 \%) & 39 (36.8\%) & - & 0.241 \\
\hline Diabetes & $13(12.3 \%)$ & $14(13.2 \%)$ & 19 (17.9\%) & - & 0.443 \\
\hline Hyperlipidemia & $33(31.1 \%)$ & $42(39.6 \%)$ & $34(32.1 \%)$ & - & 0.236 \\
\hline $\mathrm{LAD}(\mathrm{mm})$ & $28.72 \pm 5.62$ & $30.51 \pm 7.57$ & $32.11 \pm 5.15$ & 7.956 & $<0.001$ \\
\hline LVEF (\%) & $67.81 \pm 4.27$ & $67.61 \pm 4.92$ & $67.08 \pm 5.2$ & 0.657 & 0.519 \\
\hline LVEDV (ml) & $101.03 \pm 17.3$ & $103.46 \pm 18.97$ & 105.718 .91 & 1.708 & 0.183 \\
\hline LVESV (ml) & $32.36 \pm 6.35$ & $33.75 \pm 9.14$ & $35.06 \pm 9.78$ & 2.622 & 0.074 \\
\hline LVMI $\left(\mathrm{g} / \mathrm{m}^{2}\right)$ & $83.57 \pm 14.77$ & $81.03 \pm 23.78$ & $84.03 \pm 22.36$ & 0.585 & 0.055 \\
\hline IVS (mm) & $9.89 \pm 7.95$ & $9.53 \pm 1.44$ & $9.88 \pm 1.33$ & 0.198 & 0.028 \\
\hline PWT (mm) & $9.84 \pm 7.93$ & $9.26 \pm 1.37$ & $9.81 \pm 1.11$ & 0.522 & 0.059 \\
\hline$E(\mathrm{~cm} / \mathrm{s})$ & $69.27 \pm 15.13$ & $62.3 \pm 17.88$ & $61.69 \pm 15.39$ & 7.42 & 0.001 \\
\hline DT (ms) & $196.88 \pm 32.98$ & $208.7 \pm 43$ & $214.72 \pm 49.06$ & 4.211 & 0.016 \\
\hline IVRT (ms) & $87.14 \pm 13.58$ & $90.91 \pm 14.85$ & $92.44 \pm 16.49$ & 3.588 & 0.028 \\
\hline$E / A$ & $1.18 \pm 0.37$ & $1.04 \pm 0.47$ & $0.94 \pm 0.24$ & 7.927 & $<0.001$ \\
\hline $\mathrm{S}^{\prime}(\mathrm{cm} / \mathrm{s})$ & $7.54 \pm 1.71$ & $7.24 \pm 1.5$ & $7.11 \pm 1.43$ & 2.183 & 0.114 \\
\hline $\mathrm{E}^{\prime}(\mathrm{cm} / \mathrm{s})$ & $8.77 \pm 2.06$ & $7.68 \pm 2.17$ & $6.75 \pm 1.6$ & 28.32 & $<0.001$ \\
\hline$E / E^{\prime}$ & $7.17 \pm 2.21$ & $7.9 \pm 4.03$ & $8.49 \pm 3.19$ & 5.885 & 0.003 \\
\hline Diastolic dysfunction ${ }^{a}$ & $52(49.1 \%)$ & $77(72.6 \%)$ & $89(84 \%)$ & - & $<0.001$ \\
\hline Dias-D (ms) & $21.42 \pm 15.27$ & $26.42 \pm 22.18$ & $32.06 \pm 25.39$ & 2.258 & 0.001 \\
\hline Sys-D (ms) & $28.21 \pm 21.42$ & $31.79 \pm 27.6$ & $33.18 \pm 29.09$ & 1.838 & 0.161 \\
\hline \multirow[t]{2}{*}{ TPAF, ml } & Q1 $(n=106)$ & Q2 $(n=106)$ & Q3 $(n=106)$ & $\mathrm{F}$ & $p$-value \\
\hline & $<5.71 \mathrm{ml}$ & $5.71-8.53 \mathrm{ml}$ & $>8.53 \mathrm{ml}$ & & \\
\hline Age (years) & $52.74 \pm 9.37$ & $53.53 \pm 9.88$ & $54.42 \pm 10.17$ & 0.749 & 0.474 \\
\hline Male & 42 (39.6 \%) & 74 (69.8 \%) & 85 (80.2 \%) & - & $<0.001$ \\
\hline $\mathrm{BMI}\left(\mathrm{kg} / \mathrm{m}^{2}\right)$ & $24.9 \pm 3.72$ & $25.42 \pm 3.46$ & $25.31 \pm 3.74$ & 0.41 & 0.385 \\
\hline $\mathrm{SBP}(\mathrm{mmHg})$ & $119.87 \pm 17.14$ & $125.48 \pm 17.4$ & $127.27 \pm 15.51$ & 4.259 & 0.015 \\
\hline $\mathrm{DBP}(\mathrm{mmHg})$ & $75.21 \pm 11.07$ & $78.9 \pm 10.21$ & $75.95 \pm 10.13$ & 1.876 & 0.155 \\
\hline HbA1c (\%) & $5.96 \pm 0.78$ & $5.98 \pm 0.64$ & $6.08 \pm 0.70$ & 1.141 & 0.321 \\
\hline Cholesterol (mg/dL) & $201.56 \pm 36.87$ & $204.7 \pm 39.09$ & $203.56 \pm 41.8$ & 0.133 & 0.876 \\
\hline $\mathrm{TG}(\mathrm{mg} / \mathrm{dL})$ & $135.06 \pm 77.37$ & $146.32 \pm 82.82$ & $151.18 \pm 100.5$ & 0.843 & 0.431 \\
\hline
\end{tabular}


Table 1 Baseline demographics, biomarkers and echocardiographic parameters categorized by pericardial and periaortic fat volumes (Continued)

\begin{tabular}{|c|c|c|c|c|c|}
\hline $\mathrm{HDL}(\mathrm{mg} / \mathrm{dL})$ & $52.75 \pm 14.88$ & $48.85 \pm 13.22$ & $48.68 \pm 13.33$ & 3.072 & 0.048 \\
\hline $\mathrm{LDL}(\mathrm{mg} / \mathrm{dL})$ & $129.99 \pm 33.17$ & $135.68 \pm 34.56$ & $132.64 \pm 32.96$ & 0.368 & 0.68 \\
\hline $\mathrm{Hs}-\mathrm{CRP}(\mathrm{mg} / \mathrm{dL})$ & $0.167 \pm 0.022$ & $0.216 \pm 0.049$ & $0.312 \pm 0.047$ & 1.761 & 0.174 \\
\hline BNP $(p g / m L)$ & $26.41 \pm 3.93$ & $26.84 \pm 5.17$ & $38.89 \pm 8.18$ & 1.381 & 0.253 \\
\hline Hypertension & 25 (23.6 \%) & 39 (36.8 \%) & 38 (35.8 \%) & - & 0.064 \\
\hline Diabetes & 11 (10.4 \%) & $17(16 \%)$ & $18(17 \%)$ & - & 0.321 \\
\hline Hyperlipidemia & 36 (33.9 \%) & 35 (33 \%) & 38 (35.8 \%) & - & 0.908 \\
\hline $\mathrm{LAD}(\mathrm{mm})$ & $28.65 \pm 6.55$ & $30.3 \pm 6.54$ & $32.39 \pm 5.32$ & 9.765 & $<0.001$ \\
\hline LVEF (\%) & $68.23 \pm 4.05$ & $67.14 \pm 5.09$ & $67.13 \pm 5.16$ & 1.851 & 0.159 \\
\hline LVEDV (ml) & $99.98 \pm 17.88$ & $105.13 \pm 17.79$ & $105.08 \pm 19.34$ & 2.755 & 0.065 \\
\hline LVESV (ml) & $31.8 \pm 7.5$ & $34.63 \pm 8.27$ & $34.74 \pm 9.64$ & 4.059 & 0.018 \\
\hline LVMI $\left(\mathrm{g} / \mathrm{m}^{2}\right)$ & $82.11 \pm 17.22$ & $85.86 \pm 21.18$ & $80.69 \pm 22.74$ & 1.663 & 0.191 \\
\hline IVS (mm) & $9.04 \pm 1.49$ & $9.48 \pm 1.01$ & $10.79 \pm 7.88$ & 4.048 & 0.018 \\
\hline PWT (mm) & $8.93 \pm 1.11$ & $9.4 \pm 1.3$ & $10.58 \pm 7.87$ & 3.576 & 0.029 \\
\hline$E(\mathrm{~cm} / \mathrm{s})$ & $68.78 \pm 19.08$ & $65.44 \pm 15.23$ & $59.13 \pm 13.31$ & 9.465 & $<0.001$ \\
\hline DT (ms) & $198 \pm 39.47$ & $204.14 \pm 38.78$ & $218.22 \pm 47.3$ & 4.204 & 0.016 \\
\hline IVRT (ms) & $89.77 \pm 14.71$ & $89.82 \pm 13.85$ & $90.9 \pm 16.84$ & 0.199 & 0.697 \\
\hline $\mathrm{E} / \mathrm{A}$ & $1.18 \pm 0.46$ & $1.06 \pm 0.36$ & $0.92 \pm 0.28$ & 12.42 & $<0.001$ \\
\hline $\mathrm{S}^{\prime}(\mathrm{cm} / \mathrm{s})$ & $7.21 \pm 1.52$ & $7.38 \pm 1.59$ & $7.31 \pm 1.57$ & 0.328 & 0.072 \\
\hline $\mathrm{E}^{\prime}(\mathrm{cm} / \mathrm{s})$ & $8.39 \pm 2.34$ & $7.92 \pm 1.95$ & $6.89 \pm 1.78$ & 15.01 & $<0.001$ \\
\hline$E / E^{\prime}$ & $7.89 \pm 4.05$ & $7.61 \pm 2.49$ & $8.06 \pm 3.08$ & 0.921 & 0.399 \\
\hline Diastolic dysfunction ${ }^{a}$ & $59(55.7 \%)$ & 70 (66 \%) & $89(84 \%)$ & - & $<0.001$ \\
\hline Dias-D (ms) & $23.68 \pm 18.89$ & $27.21 \pm 18.75$ & $32.99 \pm 25.63$ & 3.263 & 0.003 \\
\hline Sys-D (ms) & $32.64 \pm 25.35$ & $28.49 \pm 26.43$ & $32.06 \pm 27.01$ & 1.947 & 0.165 \\
\hline
\end{tabular}

Data are presented as mean \pm standard deviation or number (percentage)

${ }^{\text {a }}$ Diastolic dysfunction was determined according to EAE/ASE recommendations [19]

Abbreviations: $P C F$ pericardial fat, TPAF thoracic periaortic fat $B M I$ body-mass-index, $S B P$ systolic blood pressure, $D B P$ diastolic blood pressure, $T G$ triglyceride, $L D L$ low-density lipoprotein, $H D L$ high-density lipoprotein, $H s$-CRP high-senstivity C-reactive protein, $B N P$ brain natriuretic peptide, $L V E F$ left ventricular ejection fraction, LVEDV left ventricular end-diastolic volume, LVESV left ventricular end-systolic volume, LAD left atrial dimension (M-mode), LVMI left ventricular mass index, IVS interventricular septum thickness (M-mode), PWT posterior wall thickness (M-mode), $E$ early mitral inflow velocity, $A$ late mitral inflow velocity, DT deceleration time, IVRT isovolumetric relaxation time, $S^{\prime}$ systolic tissue Doppler velocity, $E^{\prime}$ early-diastolic tissue Doppler velocity, Dias-D E' time-to-peak difference (diastolic dyssynchrony), Sys-D S' time-to-peak difference (systolic dyssynchrony)

control of multivariables including age, gender, BMI, systolic blood pressure, LV mass index and clinical histories (hypertension, diabetes, hyperlipidemia, smoking habits), both greater PCF and TPAF remained significantly associated with increased LAD, lower E/A ratio, reduced E' velocity and elevated E/E' ratio (all $p<0.01$ ).

\section{The association between visceral adiposity and dyssynchrony}

There were 28 subjects with abnormally high diastolic dyssynchrony (Dias-D $\geqq 55 \mathrm{~ms}$ ) and 22 subjects with systolic dyssynchrony (Sys- $\mathrm{D} \geqq 60 \mathrm{~ms}$ ). As seen in Table 1, both higher PCF and TPAF were positively associated with higher degree of diastolic dyssynchrony across tertile groups (both $p<0.01$ ). In Fig. 1e and $f$, linear regression plots also showed excellent correlation between both adipose tissue volumes and diastolic dyssynchrony (both $p<0.001$ ). In the uniand multi-variate regression models of Table 3 , we consistently demonstrated the association between both visceral adiposities and diastolic dyssynchrony (adjusted B coefficient: 0.197 \& 1.367 for PCF \& TPAF, respectively, both $p<0.001$ ).

Finally in ROC analysis, both PCF and TPAF had optimal AUROC for identifying diastolic dyssynchrony (0.736 \& $0.730,95$ \% CI: $0.648-0.824 \& 0.621-0.838$ for PCF \& TPAF, respectively, both $p<0.001)$, when defined by the aforementioned criteria (Dias-D $\geqq 55 \mathrm{~ms}$ ). PCF and TPAF set at $81.68 \& 8.11 \mathrm{ml}$ yielded the largest sensitivity and specificity (78.6 and $60 \%$ for PCF, 75 and $66.6 \%$ for TPAF, respectively) in predicting abnormally high LV diastolic dyssynchrony (Figure $2 \mathrm{c}$ and $\mathrm{d}$ ). 
Table 2 Baseline demographics, biomarkers and echocardiographic parameters of patients with and without diastolic dysfunction ${ }^{a}$

\begin{tabular}{|c|c|c|c|c|}
\hline & Normal $(n=100)$ & Diastolic dysfunction $(n=218)$ & $\mathrm{F}$ & $p$-value \\
\hline Age (years) & $50.82 \pm 9.34$ & $54.83 \pm 9.8$ & 11.85 & 0.001 \\
\hline Male & $60(60 \%)$ & $141(64.7 \%)$ & - & 0.453 \\
\hline PCF (ml) & $67.32 \pm 31.95$ & $86.79 \pm 31.77$ & 25.68 & $<0.001$ \\
\hline TPAF $(\mathrm{ml})$ & $6.32 \pm 3.73$ & $8.63 \pm 4.61$ & 19.24 & $<0.001$ \\
\hline $\mathrm{BMI}\left(\mathrm{kg} / \mathrm{m}^{2}\right)$ & $25.05 \pm 3.78$ & $25.36 \pm 4.14$ & 0.415 & 0.52 \\
\hline $\mathrm{HbA1c}(\%)$ & $4.84 \pm 2.44$ & $4.84 \pm 2.48$ & $<0.001$ & 0.992 \\
\hline Cholesterol (mg/dL) & $195.77 \pm 45.85$ & $203.94 \pm 42.61$ & 2.399 & 0.122 \\
\hline LDL (mg/dL) & $124.72 \pm 42.02$ & $124.98 \pm 46.85$ & 0.002 & 0.963 \\
\hline Hs-CRP (mg/dL) & $0.116 \pm 0.039$ & $0.166 \pm 0.034$ & 1.323 & 0.251 \\
\hline $\mathrm{BNP}(\mathrm{pg} / \mathrm{mL})$ & $21.03 \pm 4.91$ & $35.16 \pm 6.7$ & 3.572 & 0.06 \\
\hline Hypertension & $29(29 \%)$ & $73(33.5 \%)$ & - & 0.441 \\
\hline Diabetes & $12(12 \%)$ & $34(15.6 \%)$ & - & 0.493 \\
\hline Hyperlipidemia & $40(40 \%)$ & $69(31.7 \%)$ & - & 0.162 \\
\hline LAD (mm) & $28.76 \pm 6.59$ & $31.22 \pm 6.08$ & 10.64 & 0.001 \\
\hline$E(\mathrm{~cm} / \mathrm{s})$ & $68.81 \pm 15.22$ & $62.24 \pm 16.23$ & 11.7 & 0.001 \\
\hline DT (ms) & $199.95 \pm 35.88$ & $210.04 \pm 45.27$ & 3.85 & 0.045 \\
\hline IVRT (ms) & $85.66 \pm 13.66$ & $92.41 \pm 15.49$ & 13.98 & $<0.001$ \\
\hline $\mathrm{E}^{\prime}(\mathrm{cm} / \mathrm{s})$ & $10 \pm 1.59$ & $6.7 \pm 1.42$ & 344.91 & $<0.001$ \\
\hline$E / E^{\prime}$ & $7.04 \pm 1.85$ & $9.63 \pm 3.09$ & 60.51 & $<0.001$ \\
\hline Dias-D (ms) & $22.62 \pm 18.2$ & $30.42 \pm 21.66$ & 9.793 & 0.002 \\
\hline
\end{tabular}

Data are presented as mean \pm standard deviation or number (percentage)

aiastolic dysfunction was determined according to EAE/ASE recommendations [19]

Abbreviations same as Table 1

\section{Discussion}

In the present study, we observed that both pericardial and peri-aortic fat accumulations were significantly associated with diastolic dysfunction and dyssynchrony independent of LV mass and traditional risk factors. This association was slightly tighter with pericardial fat. We also identified their respective cutoff values for predicting diastolic dysfunction and dyssynchrony. To our knowledge, this is the first study to explore the relationship between such visceral adiposities and dyssynchrony by tissue Doppler imaging.

Our team has reported previously that pericardial fat was independently associated with metabolic derangements, fatty liver disease and systemic inflammation [14]. Although in this study, the association between pericardial fat and baseline metabolic risk factors was mostly weak. This may be explained by the relatively small sample size and benign clinical status of our participants. There was also a lack of correlation with BMI. Another bivariate analysis between BMI and E/E' showed no significant correlation either $(r=0.045, p=0.423)$, which is contrary to most worldwide epidemiologic studies. One possible explanation of our unusual findings might be that our average BMI is relatively normal (only 28 subjects had BMI > 30). On the other hand, the strong correlation that we demonstrated with advanced tissue Doppler metrics suggested a specific process linking pericardial fat to subclinical diastolic dysfunction that might be initiated much earlier than systemic metabolic derangements or excessive body mass accumulation. The specific effects of pericardial fat on cardiac structure and function via various mechanisms have been proposed in previous literature [22]. Compression of the heart by this enveloping fat deposit may also cause impaired LV diastolic filling, leading to atrial remodeling and dilation [17]. Pericardial fat also contains high levels of pro-atherogenic cytokines [23] that may induce inflammation and collagen turnover, leading to ventricular stiffness and diastolic dysfunction [24, 25].

Besides LA enlargement, we also observed a slight but significant increase in LV wall thickness among subjects with larger PCF or TPAF. Although diastolic dysfunction or dyssynchrony is not uncommon in people with normal wall thickness, LV hypertrophy and hypertensive heart disease are indeed the most important causes of HFpEF $[26,27]$. Therefore, it is reasonable that these changes can coexist with visceral fat accumulation and diastolic dysfunction or dyssynchrony. On the other hand, no significant difference was found with LV mass index, and multivariate regression analysis also showed that LV mass 

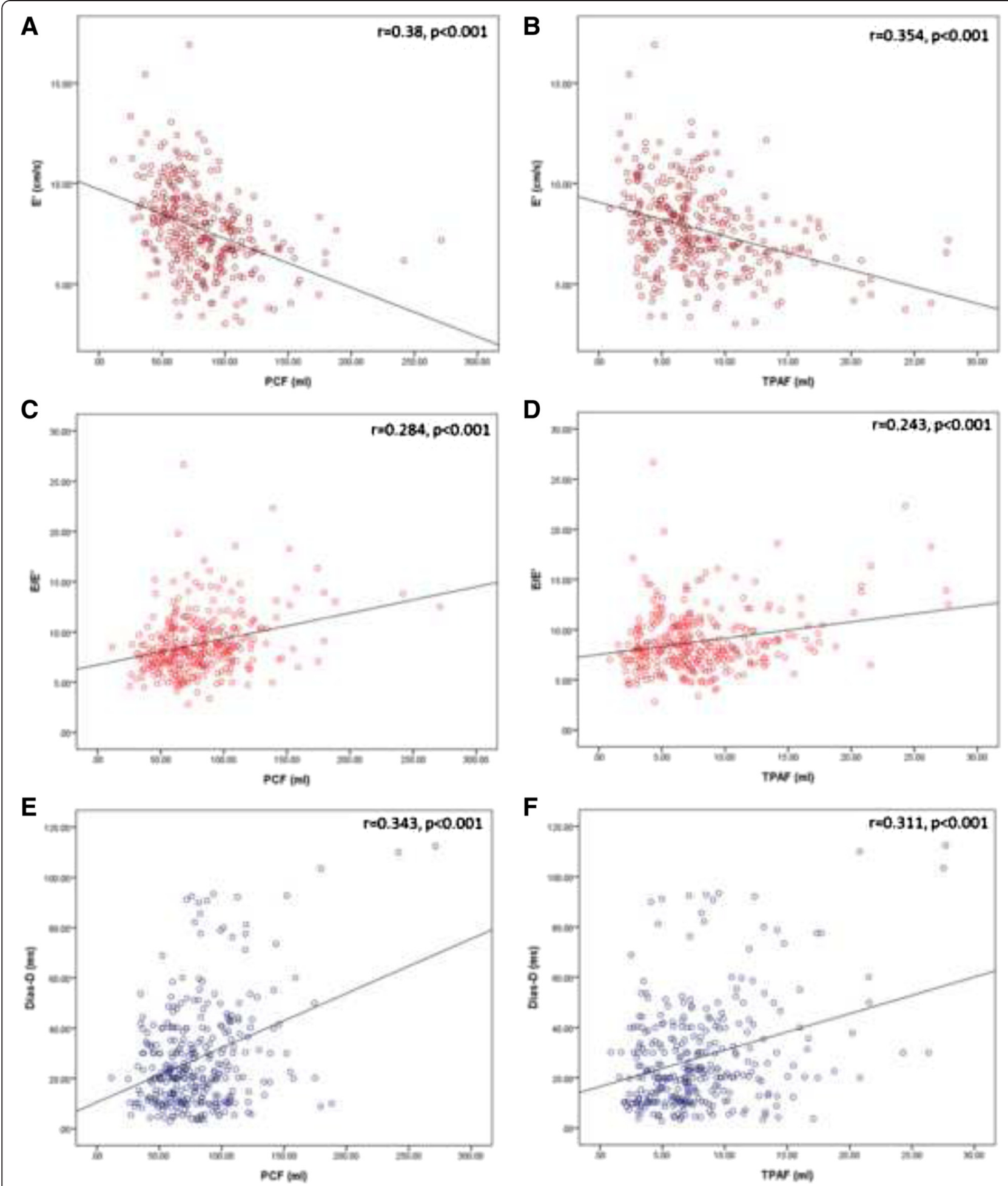

Fig. 1 Linear regression scatter plots between $E^{\prime}$ and PCF (a), TPAF (b), E/E' and PCF (c), TPAF (d), Dias-D and PCF (e), TPAF (f). All plots showed excellent statistical significance $(p<0.001)$. Abbreviations: $P C F=$ pericardial fat, TPAF $=$ thoracic periaortic fat, $E=$ early mitral inflow velocity, $\mathrm{E}^{\prime}=$ early-diastolic tissue Doppler velocity, Dias-D $=\mathrm{E}^{\prime}$ time-to-peak difference (diastolic dyssynchrony) 


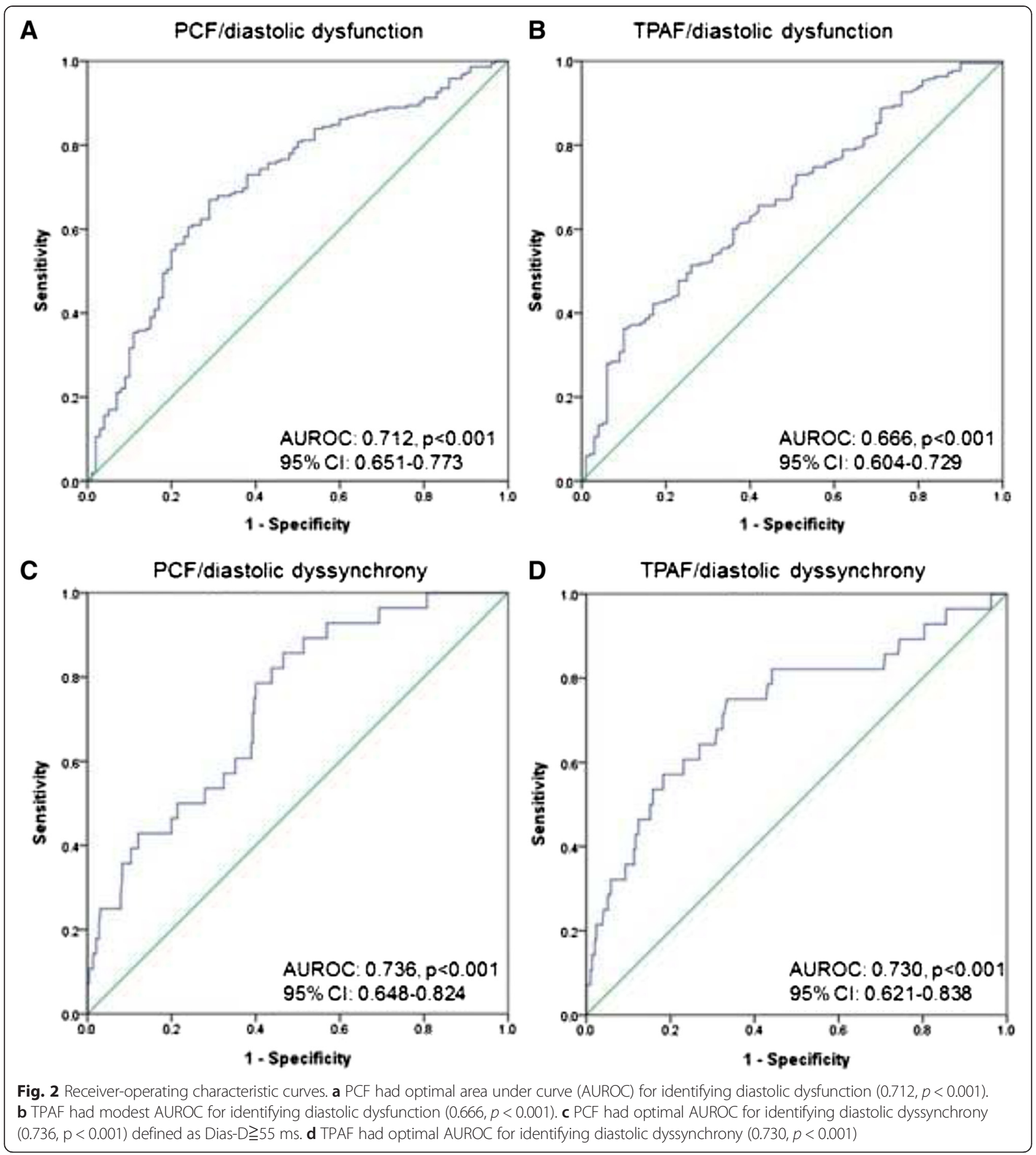

index was a nonfactor, indicating that our primary findings were independent of LV hypertrophy.

Recently, it has been discovered that dyssynchrony is not as uncommon as previously assumed, and can occur to some extent even in the normal heart [28]. Due to its excellent temporal resolution, TDI has been widely utilized for quantifying the degree of intra-ventricular mechanical dyssynchrony. Through assessment of systolic myocardial velocity, TDI has also demonstrated the presence of subclinical systolic dysfunction in HFpEF [29], hypertensive heart disease and left ventricular hypertrophy [30].

As previously mentioned, both systolic and diastolic dyssynchrony have been found to exist in HFpEF, though diastolic dyssynchrony does seem to be more prevalent and may occur exclusively $[3,4]$. This is evident with the sole presence of diastolic dyssynchrony in our study 
Table 3 Univariate and multivariate regression analyses of pericardial and periaortic fat volumes associated with echocardiographic parameters

\begin{tabular}{|c|c|c|c|c|c|c|}
\hline \multirow[t]{2}{*}{ PCF } & \multicolumn{3}{|l|}{ Univariate model } & \multicolumn{3}{|l|}{ Multivariate model $^{a}$} \\
\hline & B Coefficient (unstandardized) & $95 \% \mathrm{Cl}$ & $p$-value & B Coefficient (unstandardized) & $95 \% \mathrm{Cl}$ & $p$-value \\
\hline LVEF & -0.008 & $-0.025-0.009$ & 0.376 & -0.003 & $-0.02-0.013$ & 0.699 \\
\hline LAD & 0.043 & $0.021-0.065$ & $<0.001$ & 0.04 & $0.018-0.063$ & $<0.001$ \\
\hline E & -0.015 & $-0.07-0.04$ & 0.035 & -0.021 & $-0.077-0.036$ & 0.047 \\
\hline DT & 0.197 & $0.049-0.346$ & 0.009 & 0.148 & $-0.002-0.298$ & 0.052 \\
\hline IVRT & 0.067 & $0.015-0.12$ & 0.013 & 0.063 & $0.008-0.117$ & 0.025 \\
\hline$E / A$ & -0.002 & $-0.003--0.001$ & 0.003 & -0.002 & $-0.003--0.001$ & 0.006 \\
\hline$E^{\prime}$ & -0.024 & $-0.03--0.017$ & $<0.001$ & -0.02 & $-0.027--0.013$ & $<0.001$ \\
\hline$S^{\prime}$ & -0.006 & $-0.012--0.001$ & 0.021 & -0.005 & $-0.01-0.001$ & 0.084 \\
\hline$E / E^{\prime}$ & 0.026 & $0.016-0.036$ & $<0.001$ & 0.02 & $0.011-0.03$ & $<0.001$ \\
\hline Dias-D & 0.211 & $0.142-0.279$ & $<0.001$ & 0.197 & $0.128-0.266$ & $<0.001$ \\
\hline Sys-D & 0.086 & $0.001-0.174$ & 0.054 & 0.076 & $-0.014-0.166$ & 0.096 \\
\hline \multicolumn{7}{|l|}{ TPAF } \\
\hline & \multicolumn{3}{|c|}{ Univariate model } & \multicolumn{3}{|c|}{ Multivariate model $^{a}$} \\
\hline & B Coefficient (unstandardized) & $95 \% \mathrm{Cl}$ & $p$-value & B Coefficient (unstandardized) & $95 \% \mathrm{Cl}$ & $p$-value \\
\hline LVEF & -0.045 & $-0.172-0.082$ & 0.487 & -0.007 & $-0.131-0.117$ & 0.914 \\
\hline LAD & 0.377 & $0.215-0.539$ & $<0.001$ & 0.368 & $0.204-0.532$ & $<0.001$ \\
\hline E & -0.283 & $-0.692-0.127$ & 0.175 & -0.335 & $-0.754-0.085$ & 0.118 \\
\hline DT & 2.068 & $0.973-3.163$ & $<0.001$ & 1.712 & $0.606-2.819$ & 0.003 \\
\hline IVRT & 0.396 & $0.001-0.791$ & 0.05 & 0.351 & $-0.056-0.758$ & 0.091 \\
\hline E/A & -0.017 & $-0.026--0.009$ & $<0.001$ & -0.017 & $-0.026--0.008$ & $<0.001$ \\
\hline$E^{\prime}$ & -0.164 & $-0.216--0.111$ & $<0.001$ & -0.137 & $-0.188--0.086$ & $<0.001$ \\
\hline$S^{\prime}$ & -0.04 & $-0.081-0.001$ & 0.05 & -0.028 & $-0.069-0.012$ & 0.173 \\
\hline$E / E^{\prime}$ & 0.176 & $0.104-0.249$ & $<0.001$ & 0.134 & $0.064-0.203$ & $<0.001$ \\
\hline Dias-D & 1.461 & $0.947-1.976$ & $<0.001$ & 1.367 & $0.848-1.885$ & $<0.001$ \\
\hline Sys-D & 0.489 & $-0.165-1.143$ & 0.142 & 0.415 & $-0.258-1.088$ & 0.225 \\
\hline
\end{tabular}

${ }^{a}$ Adjusted for age, gender, BMI, systolic blood pressure, LV mass index and clinical histories (hypertension, diabetes, hyperlipidemia, smoking habits) Abbreviations same as Table 1

population. Several possible mechanisms have been suggested. First, early diastolic filling is mainly determined by the coordination of its previous systolic phase. Discordance of systolic contraction will lead to prolonged relaxation and reduced diastolic filling time [31]. Second, increased afterload may contribute to diastolic dyssynchrony through elevated wall stress and myocardial oxygen demand, leading to uneven distributions in coronary blood flow [32, 33]. Dyssynchrony has been reported to be associated with LV filling pressure in asymptomatic hypertensive patients with normal QRS duration and EF [34]. Other etiologies include conduction system disease, hypertrophy or fibrosis.

One recent study using strain imaging showed that obesity is a significant independent predictor of intraventricular dyssynchrony [35]. Another TDI-related study showed significant reductions in myocardial velocities, global and regional strain among obese subjects [36]. There has been growing interest in the potentially toxic effects of "cardiac steatosis" (excessive deposition of triglycerides in the myocardial cells) [37], which may induce myocardial fibrosis, cellular apoptosis and mitochondrial dysfunction via free fatty acid turnover process $[38,39]$. Cardiac steatosis may also be an independent predictor of diastolic dysfunction in diabetic patients [40].

Due to its proximity to the myocardium and the same coronary blood supply, pericardial fat may have stronger correlations with coronary vasculopathy [41], cardiac structure and function than other measures of adiposity, such as total visceral fat or subcutaneous fat tissue [42]. The current study results suggest that visceral fat adjacent to cardiac structures may have an independent role in mediating regional disturbances of coronary 
supply and cardiac steatosis, resulting in the development of intra-ventricular dyssynchrony. Additionally, the independent association between pericardial fat burden and increased LA dimension, an important clinical feature and predictor of HF mechanics beyond ventricular hypertrophy [43], further highlighted the possible role of regional adipose depots in mediating HF development.

\section{Limitations}

There are several limitations in our study. The first, our study has a male gender predominance, which may be somewhat biased. Secondly, this survey is retrospective and cross-sectional, without longitudinal follow up or validation with clinical outcomes. Thirdly, our data come from asymptomatic Asian participants who underwent primary cardiovascular health survey, and who therefore may not be fully representative of the broader general population in daily out-patient clinics. Finally, only basal septal and lateral segments were used to calculate dyssynchrony, which may be an overly simplistic estimate. We also acknowledge that our data regarding LA remodeling were mainly focused on LA diameter rather than 2-dimensional echo-based volume measurements.

\section{Conclusion}

Our study provides valuable insights into the underlying of potential pathophysiologic mechanisms that visceral adipose tissue may exert several biological effects on myocardial contractile or diastolic functions and coordination, leading to impaired diastolic dysfunction, more elevated filling pressures and prolonged dyssynchrony, even in asymptomatic subjects. Our findings are critical in the conceptual framework of current understanding in obesity or metabolic derangements related myocardial asynergy and predisposition to preserved ejection fraction HF development. Excessive visceral adiposity burden linked to metabolic derangements, when assessed by CT, may thus serve as a possible marker or target for associated diagnostic purposes and future therapeutic intervention in these population.

\section{Additional files}

Additional file 1: Figure S1. Pulsed-wave tissue Doppler measurement of intra-ventricular dyssynchrony. Upper panel: lateral wall TDI waveform. Lower panel: medial wall TDI waveform. The time intervals between QRS onset and peak of S'/E' were measured respectively. Systolic dyssynchrony was presented as the absolute time-to-peak difference of $S^{\prime}$ between lateral and medial segments (T1-T3). Diastolic dyssynchrony was presented as the absolute time-to-peak difference of $\mathrm{E}^{\prime}$ (T2-T4). Abbreviations: $\mathrm{SI}=$ lateral systolic myocardial velocity, El = lateral early-diastolic myocardial velocity, $\mathrm{Sm}=$ medial systolic myocardial velocity, $\mathrm{Em}=$ medial early-diastolic myocardial velocity. (TIFF $799 \mathrm{~kb}$ )

Additional file 2: Figure S2. 3D-reconstruction of total pericardial fat volume from axial, sagittal and coronal images. Pericardial fat (yellow color) was selected as all adipose tissue within the pericardial sac and subtracted from the adjacent cardiac structures. (A) A case with large pericardial fat (total volume $=174.3 \mathrm{ml}$ ). This subject has decreased diastolic tissue velocity (lateral $\mathrm{E}^{\prime}=4 \mathrm{~cm} / \mathrm{s}$ ) and prolonged diastolic dyssynchrony (80 ms). (B) A case with small pericardial fat (total volume $=26.39 \mathrm{ml}$ ). This subject has normal diastolic tissue velocity (lateral $E^{\prime}=12 \mathrm{~cm} / \mathrm{s}$ ) and diastolic dyssynchrony (10 ms). (ZIP $1204 \mathrm{~kb}$ )

\section{Abbreviations}

HF: Heart failure; HFpEF: Heart failure with preserved ejection fraction; LV: Left ventricular; PCF: Pericardial fat; E: Early mitral inflow velocity; A: Late mitral inflow velocity; DT: Deceleration time; IVRT: Isovolumetric relaxation time; TDI: Tissue Doppler imaging; S': Systolic tissue Doppler velocity; E': Early-diastolic tissue Doppler velocity; Sys-D: $S^{\prime}$ time-to-peak difference (systolic dyssynchrony); Dias-D: E' time-to-peak difference (diastolic dyssynchrony); MDCT: Multidetector computed tomography; TPAF: Thoracic periaortic fat.

\section{Competing interests}

The authors declare that they have no competing interests.

\section{Authors' contributions}

YHL, CJH participated in study design, performed the echocardiographic measurements and drafted the manuscript. CHY performed the CT measurements. KTS performed statistical analysis. CHS, TCH, HIY provided assistance in literature review. TSW, FSY, HGB provided assistance in CT measurement and method standardization. CLH conceived of this study, participated in its design and coordination and revised the manuscript. All authors read and approved the final manuscript.

\section{Acknowledgements}

This work was in part supported in part by grants from the National Science Council (NSC 103-2314-B-010-005-MY3, 103-2314-B-195-001-MY3, 101-2314-B195-020-MY1, MOST 103-2314-B-195-006-MY3), and Mackay Memorial Hospital (10271, 10248, 10220, 10253, 10375, 10358, E-102003).

\section{Author details}

'Division of Cardiology, Department of Internal Medicine, Mackay Memorial Hospital, No. 92, Sec. 2, Zhongshan N. Road, Taipei, Taiwan. ${ }^{2}$ Division of Cardiology, Department of Internal Medicine, Mackay Memorial Hospital, Hsinchu, Taiwan. ${ }^{3}$ Mackay Junior College of Medicine, Nursing, and Management, Taipei, Taiwan. ${ }^{4}$ Mackay Medical College, Taipei, Taiwan. ${ }^{5}$ Department of Radiology, Mackay Memorial Hospital, Taipei, Taiwan. ${ }^{6}$ Department of Biomedical Imaging and Radiological Sciences, National Yang Ming University, Taipei, Taiwan. ${ }^{7}$ The Institute of Health Policy and Management, College of Public Health, National Taiwan University, Taipei, Taiwan. ${ }^{8}$ University Hospitals Harrington Heart \& Vascular Institute, Division of Cardiology, Case Western Reserve University, Cleveland, OH, USA.

Received: 26 June 2015 Accepted: 26 October 2015

Published online: 30 October 2015

\section{References}

1. Thom T, Haase N, Rosamond W, Howard VJ, Rumsfeld J, Manolio T, et al. Heart disease and stroke statistics_-2006 update: a report from the American Heart Association Statistics Committee and Stroke Statistics Subcommittee. Circulation. 2006;113:e85-e151.

2. Shah SJ, Gheorghiade M. Heart failure with preserved ejection fraction: treat now by treating comorbidities. JAMA. 2008;300:431-3.

3. Wang J, Kurrelmeyer KM, Torre-Amione G, Nagueh SF. Systolic and diastolic dyssynchrony in patients with diastolic heart failure and the effect of medical therapy. J Am Coll Cardiol. 2007;49:88-96.

4. Yu CM, Zhang Q, Yip GWK, Lee PW, Kum LC, Lam YY, et al. Diastolic and systolic asynchrony in patients with diastolic heart failure: a common but ignored condition. J Am Coll Cardiol. 2007;49:97-105.

5. Kane GC, Karon BL, Mahoney DW, Redfield MM, Roger VL, Burnett Jr JC, et al. Progression of left ventricular diastolic dysfunction and risk of heart failure. JAMA. 2011;306:856-63.

6. Ingelsson E, Sundström J, Arnlöv J, Zethelius B, Lind L. Insulin resistance and risk of congestive heart failure. JAMA. 2005;294:334-41. 
7. Suzuki T, Katz R, Jenny NS, Zakai NA, LeWinter MM, Barzilay J, et al. Metabolic syndrome, inflammation, and incident heart failure in the elderly: the cardiovascular health study. Circ Heart Fail. 2008;1:242-8.

8. Bahrami H, Bluemke DA, Kronmal R, Bertoni AG, Lloyd-Jones DM, Shahar E, et al. Novel metabolic risk factors for incident heart failure and their relationship with obesity: the MESA (Multi-Ethnic Study of Atherosclerosis) study. J Am Coll Cardiol. 2008;51:1775-83.

9. Janssen I, Heymsfield SB, Allison DB, Kotler DP, Ross R. Body mass index and waist circumference independently contribute to the prediction of nonabdominal, abdominal subcutaneous, and visceral fat. Am J Clin Nutr. 2002;75:683-8.

10. Turkbey EB, McClelland RL, Kronmal RA, Burke GL, Bild DE, Tracy RP, et al. The impact of obesity on the left ventricle: the Multi-Ethnic Study of Atherosclerosis (MESA). JACC Cardiovasc Imaging. 2010;3:266-74.

11. Alpert MA, Lambert CR, Terry BE, Cohen MV, Mukerji V, Massey CV, et al. Influence of left ventricular mass on left ventricular diastolic filling in normotensive morbid obesity. Am Heart J. 1995;130:1068-73.

12. Kopelman PG. Obesity as a medical problem. Nature. 2000;404:635-43.

13. Russo C, Jin Z, Homma S, Rundek T, Elkind MS, Sacco RL, et al. Effect of obesity and overweight on left ventricular diastolic function: a communitybased study in an elderly cohort. J Am Coll Cardiol. 2011;57:1368-74.

14. Lai YH, Yun CH, Yang FS, Liu CC, Wu YJ, Kuo JY, et al. Epicardial adipose tissue relating to anthropometrics, metabolic derangements and fatty liver disease independently contributes to serum high-sensitivity C-reactive protein beyond body fat composition: a study validated with computed tomography. J Am Soc Echocardiogr. 2012;25:234-41.

15. Doesch C, Suselbeck T, Leweling H, Fluechter S, Haghi D, Schoenberg SO, et al. Bioimpedance analysis parameters and epicardial adipose tissue assessed by cardiac magnetic resonance imaging in patients with heart failure. Obesity (Silver Spring). 2010;18:2326-32.

16. lacobellis G, Ribaudo MC, Assael F, Vecci E, Tiberti C, Zappaterreno A, et al. Echocardiographic epicardial adipose tissue is related to anthropometric and clinical parameters of metabolic syndrome: a new indicator of cardiovascular risk. J Clin Endocrinol Metab. 2003;88:5163-8.

17. lacobellis G, Leonetti F, Singh N, Sharma M. Relationship of epicardial adipose tissue with atrial dimensions and diastolic function in morbidly obese subjects. Int J Cardiol. 2007;115:272-3.

18. Lang RM, Bierig M, Devereux RB, Flachskampf FA, Foster E, Pellikka PA, et al. Recommendations for chamber quantification: a report from the American Society of Echocardiography's Guidelines and Standards Committee and the Chamber Quantification Writing Group, developed in conjunction with the European Association of Echocardiography, a branch of the European Society of Cardiology. J Am Soc Echocardiogr. 2005;18:1440-63.

19. Nagueh SF, Appleton CP, Gillebert TC, Marino PN, Oh JK, Smiseth OA, et al. Recommendations for the evaluation of left ventricular diastolic function by echocardiography. J Am Soc Echocardiogr. 2009;22:107-33.

20. Shanks M, Bertini M, Delgado V, Ng AC, Nucifora G, van Bommel RJ, et al. Effect of biventricular pacing on diastolic dyssynchrony. J Am Coll Cardiol. 2010;56:1567-75.

21. Yun CH, Lin TY, Wu YJ, Liu CC, Kuo JY, Yeh HI, et al. Pericardial and thoracic peri-aortic adipose tissues contribute to systemic inflammation and calcified coronary atherosclerosis independent of body fat composition, anthropometric measures and traditional cardiovascular risks. Eur J Radiol. 2012;81:749-56

22. Sacks HS, Fain JN. Human epicardial adipose tissue: a review. Am Heart J. 2007;153:907-17.

23. Mazurek T, Zhang L, Zalewski A, Mannion JD, Diehl JT, Arafat H, et al. Human epicardial adipose tissue is a source of inflammatory mediators. Circulation. 2003;108:2460-6.

24. Martos R, Baugh J, Ledwidge M, O'Loughlin C, Conlon C, Patle A, et al. Diastolic heart failure: evidence of increased myocardial collagen turnover linked to diastolic dysfunction. Circulation. 2007;115:888-95.

25. Mak GJ, Ledwidge MT, Watson CJ, Phelan DM, Dawkins IR, Murphy NF, et al. Natural history of markers of collagen turnover in patients with early diastolic dysfunction and impact of eplerenone. J Am Coll Cardiol. 2009:54:1674-82

26. Levy D, Garrison RJ, Savage DD, Kannel WB, Castelli WP. Prognostic implications of echocardiographically determined left ventricular mass in the Framingham Heart Study. N Engl J Med. 1990;322:1561-6.
27. Koren MJ, Devereux RB, Casale PN, Savage DD, Laragh JH. Relation of left ventricular mass and geometry to morbidity and mortality in uncomplicated essential hypertension. Ann Intern Med. 1991;114:345-52.

28. Zwanenburg JJ, Gotte MJ, Kuijer JP, Heethaar RM, van Rossum AC, Marcus JT. Timing of cardiac contraction in humans mapped by high-temporal-resolution MRI tagging: early onset and late peak of shortening in lateral wall. Am J Physiol Heart Circ Physiol. 2004;286:H1872-80.

29. Yu CM, Lin H, Yang H, Kong SL, Zhang Q, Lee SW. Progression of systolic abnormalities in patients with "isolated" diastolic heart failure and diastolic dysfunction. Circulation. 2002;105:1195-201.

30. Wang M, Yip GWK, Wang A, Zhang Y, Ho PY, Tse MK, et al. Tissue Doppler imaging provides incremental prognostic value in patients with hypertension and left ventricular hypertrophy. J Hypertension. 2005;23:183-91.

31. Gibson DG, Brown DJ. Relation between diastolic left ventricular wall stress and strain in man. Br Heart J. 1974;36:1066-77.

32. Bauer M, Cheng S, Unno K, Lin FC, Liao R. Regional cardiac dysfunction and dyssynchrony in a murine model of afterload stress. PLoS One. 2013;8:e59915

33. Bonow RO, Vitale DF, Bacharach SL, Frederick TM, Kent KM, Green MV. Asynchronous left ventricular regional function and impaired global diastolic filling in patients with coronary artery disease: reversal after coronary angioplasty. Circulation. 1985;71:297-307.

34. Chang SA, Kim HK, Kim DH, Kim YJ, Sohn DW, Oh BH, et al. Left ventricular systolic and diastolic dyssynchrony in asymptomatic hypertensive patients. J Am Soc Echocardiogr. 2009;22:337-42.

35. Purushottam B, Parameswaran AC, Figueredo VM. Dyssynchrony in obese subjects without a history of cardiac disease using velocity vector imaging J Am Soc Echocardiogr. 2011;24:98-106.

36. Tumuklu MM, Etikan I, Kisacik B, Kayikcioglu M. Effect of obesity on left ventricular structure and myocardial systolic function: assessment by tissue Doppler imaging and strain/strain rate imaging. Echocardiography. 2007:24:802-9

37. Chiu HC, Kovacs A, Ford DA, Hsu FF, Garcia R, Herrero P, et al. A novel mouse model of lipotoxic cardiomyopathy. J Clin Invest. 2001;107:813-22.

38. Gastaldelli A, Morales MA, Marraccini P, Sicari R. The role of cardiac fat in insulin resistance. Curr Opin Clin Nutr Metab Care. 2012;15:523-8.

39. Christoffersen C, Bollano E, Lindegaard ML, Bartels ED, Goetze JP, Andersen CB, et al. Cardiac lipid accumulation associated with diastolic dysfunction in obese mice. Endocrinology. 2003;144:3483-90.

40. Rijzewijk LJ, van der Meer RW, Smit JW, Diamant M, Bax JJ, Hammer S, et al. Myocardial steatosis is an independent predictor of diastolic dysfunction in type 2 diabetes mellitus. J Am Coll Cardiol. 2008;52:1793-9.

41. Wang CP, Hsu HL, Hung WC, Yu TH, Chen YH, Chiu CA, et al. Increased epicardial adipose tissue (EAT) volume in type 2 diabetes mellitus and association with metabolic syndrome and severity of coronary atherosclerosis. Clin Endocrinol. 2009;70:876-82.

42. lacobellis G, Pond CM, Sharma AM. Different "weight" of cardiac and general adiposity in predicting left ventricle morphology. Obesity (Silver Spring). 2006;14:1679-84.

43. Melenovsky V, Borlaug BA, Rosen B, Hay I, Ferruci L, Morell CH, et al. Cardiovascular features of heart failure with preserved ejection fraction versus nonfailing hypertensive left ventricular hypertrophy in the urban Baltimore community: the role of atrial remodeling/dysfunction. J Am Coll Cardiol. 2007:49:198-207

\section{Submit your next manuscript to BioMed Central and take full advantage of:}

- Convenient online submission

- Thorough peer review

- No space constraints or color figure charges

- Immediate publication on acceptance

- Inclusion in PubMed, CAS, Scopus and Google Scholar

- Research which is freely available for redistribution 